both fragments differs in each variant. Has B lost its basic copy gene? This seems unlikely, because B was generated from A, which should therefore contain the basic copy of gene B. Is one of the B genes in an environment such that it is susceptible to the action of DNase I? Are both copies truly in chromosomal DNA? Answers to both questions are unknown. There appear to be four copies of gene $\mathrm{C}$, in clones $\mathrm{A}, \mathrm{B}$ and $C$. Two copies are invariant, whereas the sizes of the fragments carrying the other two genes alters in a way similar to that observed for variant antigen B.

Such results imply that different mechanisms may exist to control expression of VSG genes. The ELC model is attractive because it offers a ready explanation for control of antigenic variation, but as the principal proponents of the model themselves admit ${ }^{2,3}$, not all the data are consistent with generation of an ELC. Another problem relates to the ploidy of trypanosomes. It has been estimated that ELC genes and basic copy genes exist in a ratio of $1: 1$ (ref. 4). If this estimate is correct, and if trypanosomes are diploid as generally assumed ${ }^{13}$, this implies coordinate expression of the products of two alleles. If the estimate is incorrect, some mechanism of allelic exclusion must operate. Such problems are not resolved by incorporating the results of the ILRAD group for their findings suggest no obvious mechanism for the control of antigenic variation. The two B-specific genes identified could, for example, be allelic forms of the same antigen, but it is not apparent how expression of each is controlled. Is one copy silent, and can the other be activated? Hence the importance of the DNase I protection experiment. Perhaps a very large fragment of DNA $(>50 \mathrm{~kb})$ is transposed into a site allowing expression. Such a phenomenon could be identified only by using restriction enzymes which cut outside the transposed unit and a detection system which allowed resolution of very large fragments. All groups agree that the sequence on the 3 ' side of the active gene is unusual2,12 in that it is apparently devoid of restriction enzyme sites for up to $15 \mathrm{~kb}$, and is therefore presumably a simple repetitive sequence. The apparent clustering of many restriction enzyme sites at the end of this sequence could represent the end of the DNA molecule. Resolution of these paradoxes is awaited with interest.

As a further sophistication, VSG genes appear to be capable of localized 'somatic' hypermutation which may extend the antigenic diversity yet further. Sequence analyses of VSGs have shown that the N-terminal 350-400 amino acids are highly variable. The next 100 or so amino acids form a homology region which can be used to divide VSGs into two subsets, followed by the hydrophobic tail of about 20 amino acids which is absent from mature VSGs $^{7,8,14-16}$. Rice-Ficht et al. (see p.676) have now compared the DNA sequence of the basic copy gene with cDNA sequences

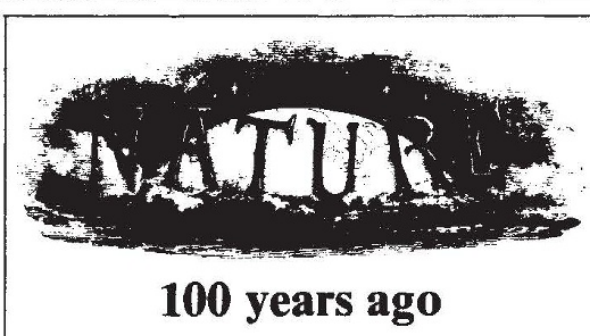

We observe that a correspondent of a daily paper proposes that men addicted to the pursuits of science should be called scientiates, after the Italian scienziati; and in like manner the studies of science, sciential studies (studj scienziali). The substitution of the American scientist for our unsatisfactory phrase men of science is of course much to be deprecated; perhaps we shall come to accept Sir William Thomson's proposed use of naturalist for the designation in question, if its sense may be extended. Scientific studies is a phrase which cannot be commended for accuracy.

A curious and little-known experiment, showing the resistance of the air in guns, is

corresponding to the mRNA transcribed from the ELC of variant antigen gene A described by Majiwa et al. Surprisingly, considerable differences were observed in the variable region and in the $\mathrm{C}$-terminal hydrophobic tail, amounting to nucleotide changes at four per cent of all codon positions. The homology region is identical in the two sequences. As previously recorded, the $\mathbf{3}^{\prime}$-non-translated sequences are quite different in the two clones, consistent with generation of the ELC through recombination in this region. What could be the significance of such findings?

One possibility, which cannot be rigorously excluded, is that the 'wrong' basic copy gene has been cloned and sequenced. A second is that cDNA cloning artefacts are responsible. However, sequences from different cDNA clones were compared and found to be identical, and cloning artefacts are unlikely to respect homology regions. The assumption must therefore be that such sequence alterations are generated in the formation of the ELC, with the possibilities for 'somatic' diversity that that implies. Changes at nine per cent of codon positions in the influenza haemagglutinin are sufficient to produce new variants, corresponding to alterations at four antigenic determinants ${ }^{17}$, hence the trypanosome may be using this mechanism to extend enormously its repertoire of VSGs.

Two difficulties remain. First, only half the VSG variable region has so far been analysed in this way. Does the $\mathrm{N}$-terminal region show similar hypermutation? Second, how much of this variable region is exposed on the trypanosome? It has been known for some time that immunizations with either living trypanosomes, or pure VSG can lead to the production of a high proportion of variant-specific monoclonal antibodies which will react with soluble VSG, but not with the surface of the described by Prof. Daniel Colladon, of Geneva. It resembles a feat that was sometimes performed by soldiers with the old Swiss carbines. M. Colladon fully charged with compressed air the hollow iron breech of an air-gun, serving as reservoir. Having screwed up the gun, he introduced a round lead ball, running freely, but nearly filling the bore; then, placing the gun vertical, he seized the upper end and pressed his thumb vigorously on the mouth. The gun was then "fired" by an assistant; the thumb remained in position, and the ball was heard to fall back in the bore. Thereupon, after recharging the breech and with the same ball, he shot the latter at a pine board about 4in. thick and it passed through. The experiment, $\mathbf{M}$. Colladon says, is without danger, if the operator is sure of the strength of his thumb, if the gun is more than $32 \mathrm{in}$. long, and if the ball is spherical and nearly fills the gun (in which it must act like a piston). While M. Colladon has repeated the experiment twenty or thirty times, without the least inconvenience a trial of it is perhaps hardly to be recommended.

From Nature 26, 353; August 10, 1882.

\section{trypanosome $\mathrm{e}^{18,19}$.}

Trypanosomes readily shed their VSG during infection, so much of the immune response must be against soluble VSG, but why have variant-specific determinants on soluble VSGs which are not exposed on the surface of the trypanosome, and which are therefore not under selective pressure? Of the several potential antigenic determinants on the VSG molecule only a small number may be exposed on the closely packed surface coat, and such sites may be 'hot spots' for the 'somatic' hypermutation described by Rice-Ficht $e t$ $a l$. For the trypanosome to then induce the host to make variant-specific antibody which cannot recognize the trypanosome surface could be a diversionary form of antigenic competition which surely would be the ultimate caprice of this virtuoso parasite?

I. Bloom, B.R. Nature 279, 21 (1979).

2. Borst, $\mathrm{P}$ et al Cold Spring Harb. Symp. quant, Biol. 45,935 (1980).

3. Hoeijmakers, J.H.J., Frach, A.C.C., Bernards, A. Borst, P. \& Cross, (i.A.M. Nature 284, 78 (1980)

4. Pays, E., Van Meirvenne, N., Le Ray, D. \& Steinert, M. Proc. natn. Acad. Sci. U.S. A. 78, 2673 (1981).

Pays, E., Lheureux, M. \& Steinert, M. Nature 292, 265 (1981).

Bernards, A. et al. Cell 27, 497 (1981)

Rice-Ficht, A.C., Chen, K.K. \& Donelson, J.E. Nature 294, 53 (1981).

8. Boothroyd, J.C., Cross, G.A.M., Hoeijmakers, J.H.J. \& Borst, P. Nature 288, 624 (1980)

9. Boothroyd, J.C.. Paynter. C.A.. Cross, G.A.M. Bernards, A. \& Borst, P. Nucleic Acids Res. 9, 4735 (1981).

10. Van der Ploeg, L.H.T., Bernards, A., Rijsewijk \& Borsı, P. Nucleix Acids Res. 10, 593 (1982).

11. Williams, R.O., Young, J.R. \& Majiwa, P.A.O. Nature 282, 847 (1979).

12. Williams, R.O., Young, J.R., Majiwa, P.A.O., Doyle, J.J. \& Shapiro, S.Z. Cold Spring Harb. Symp. quant. Biol. 45, 945 (1980).

13. Taii, A. Nature $287,536(1980)$

14. Bridgen. P.J.. Cross, G.A.M. \& Bridgen, J. Nature $263,613(1976)$

15. Holder, A.A. \& Cross, G.A.M Molec, biochem Parasit. 2, 135 (1981)

16. Matthyseens, G., Michiels, F., Hammers, R., Pays, E. \& Steinert, M. Nature 293, 230 (1981).

7. Webster, R.G., Laver, W.G.. Air. G.M. \& Schild, G.C. Nature 296. 115 (1982).

18. Pearson, T.W et al. J. immun. Meth. 34, 141 (1980)

19. Lyon, J.A., Pratt, J.M., Travis, R.W., Doctor, B.P. \& Olenick, J.G. J. Immun. 126, 134 (1981). 